\title{
Radiology in Transplant
}

\author{
Amy K. Kim • Michael L. Schilsky
}

Published online: 19 September 2014

(C) Springer International Publishing AG 2014

\begin{abstract}
There have been several advances in liver imaging that have affected liver transplantation, with the adoption of new policies for hepatocellular carcinoma (HCC) and exploration of new imaging techniques for assessment of liver lesions, steatosis, and fibrosis. The implementation of the Organ Procurement and Transplant Network (OPTN) criteria for non-invasive diagnosis of HCC impacted organ allocation, and the proposed LI-RADS (Liver Imaging Reporting and Data System) recommendations should enable standardization of the reporting of liver lesion characteristics. New methods to enhance existing magnetic resonance imaging (MRI) or computed tomography (CT) techniques have improved correlation with tumor histology and with prognosis of the disease after locoregional treatment. The use of contrast-enhanced ultrasound may also be a promising cost-efficient method for early detection of HCC. This review also comments on the use of $\mathrm{CT}$ and MRI for evaluating living donor candidates, and the use of elasticity imaging with MRI or ultrasound for graft fibrosis after liver transplantation.
\end{abstract}

Keywords OPTN HCC criteria · Hepatocellular carcinoma · Liver neoplasm - Acoustic radiation force impulse elastography $\cdot$ Living donor liver transplant $\cdot$ Magnetic resonance imaging $\cdot$ Ultrasonography $\cdot$ contrast-enhanced · Liver transplant graft fibrosis

\footnotetext{
A. K. Kim • M. L. Schilsky $(\bowtie)$

Yale New Haven Transplantation Center, Yale University Medical Center, 333 Cedar St. LMP 1080, New Haven, CT 06520, USA

e-mail: michael.schilsky@yale.edu
}

A. K. Kim

e-mail: amy.kim@yale.edu

\section{Introduction}

Methodological innovation for liver imaging and advances with hepatobiliary contrast agents that affect our ability to diagnose hepatocellular carcinoma (HCC), adoption of new policies that govern non-invasive radiological diagnosis of HCC, as well as estimation of hepatic steatosis and fibrosis dominate the advances this year with respect to liver transplantation and radiology. In addition, some of these advances in imaging have also been applied to living donor liver transplantation (LDLT). The following review captures highlights from these subjects and provides commentary as to their impact on the field of liver transplantation.

\section{Hepatocellular Carcinoma (HCC): Diagnosis}

The adoption of the Organ Procurement and Transplant Network (OPTN) criteria for non-invasive diagnosis of HCC has the highest level of impact on our current clinical practice for liver transplantation. Non-invasive diagnosis of HCC is made by identification of an arterial-phase tumor blush from contrast material in highly vascular primary liver tumors that derive their circulation from the hepatic artery, and is more accurate when radiologic features such as washout on the portal venous phase, presence of pseudocapsule, as well as lesion size $>2 \mathrm{~cm}$ and growth of the lesions are present. These additional features are now required for HCC diagnosis without liver biopsy for liver transplant patients seeking increased priority for transplant based on current UNOS (United Network for Organ Sharing) rules for liver allocation. Current rules for non-invasive $\mathrm{HCC}$ diagnosis (using minimum technical requirements for performance of imaging that were also published) are summarized in Table 1. Use of these criteria for organ allocation for patients with HCC was predicted to have a clear impact based on results of the study by Fowler et al. 
Table 1 Organ Procurement and Transplant Network criteria

\begin{tabular}{|c|c|c|}
\hline OPTN class 5 & Criteria & Eligibility \\
\hline Class 5B & $\begin{array}{l}\text { Must meet all } 3 \text { criteria } \\
\text { - Single nodule } \geq 2 \mathrm{~cm} \text { and } \leq 5 \mathrm{~cm} \\
\text { - Increased contrast enhancement on late hepatic } \\
\text { arterial phase } \\
\text { - Washout on portal venous/delayed phase OR } \\
\text { capsule/peripheral rim enhancement OR } \\
\text { growth by } \geq 50 \% \text { on serial CT or } \\
\text { MRI }<6 \text { months apart OR biopsy }\end{array}$ & Automatic MELD priority score \\
\hline Class 5A & $\begin{array}{l}\text { Must meet all } 3 \text { criteria } \\
\text { - Single nodule } \geq 1 \mathrm{~cm} \text { and } \leq 2 \mathrm{~cm} \\
\text { - Increased contrast enhancement on late hepatic } \\
\text { arterial phase } \\
\text { - Washout on portal venous/delayed phase OR } \\
\text { capsule/peripheral rim enhancement OR growth } \\
\text { by } \geq 50 \% \text { on serial CT or MRI } \leq 6 \text { months } \\
\text { apart OR biopsy }\end{array}$ & No MELD priority score \\
\hline $\begin{array}{r}\text { Class 5A-g } \\
\text { (growth) }\end{array}$ & $\begin{array}{l}\text { Growth criteria do not apply to ablated lesions } \\
\text { - Single nodule } \geq 1 \mathrm{~cm} \text { and } \leq 2 \mathrm{~cm} \\
\text { - Increased contrast enhancement on late } \\
\text { hepatic arterial phase } \\
\text { - Growth by } \geq 50 \% \text { documented on serial CT } \\
\text { or MRI } \leq 6 \text { months apart }\end{array}$ & $\begin{array}{l}\text { This alone does not permit automatic } \\
\text { MELD priority, but if another } \\
5 \mathrm{~A} \text { or } 5 \mathrm{~A}-\mathrm{g} \text { lesion found, patient } \\
\text { will be eligible }\end{array}$ \\
\hline $\begin{array}{l}\text { Class } 5 \mathrm{~T} \\
\text { (treated) }\end{array}$ & $\begin{array}{l}\text { - Past loco-regional treatment for HCC } \\
\text { (OPTN class } 5 \text { lesions or biopsy proven) } \\
\text { - Evidence of persistent/recurrent HCC seen } \\
\text { as nodular or crescentic-enhancing tissue } \\
\text { on late arterial imaging }\end{array}$ & Continued priority points \\
\hline Class $5 \mathrm{X}$ & $\begin{array}{l}\text { - Lesion diameter } \geq 5 \mathrm{~cm} \\
\text { - Increased contrast enhancement on late hepatic } \\
\text { arterial phase } \\
\text { - Washout on portal venous/delayed phase OR } \\
\text { capsule/peripheral rim enhancement }\end{array}$ & $\begin{array}{l}\text { Not eligible for automatic exception } \\
\text { points. These lesions must be } \\
\text { considered by Regional Review } \\
\text { Board }\end{array}$ \\
\hline
\end{tabular}

Based on OPTN policy as of 13 October2013 [1]

$C T$ computed tomography, $H C C$ hepatocellular carcinoma, $M E L D$ Model for End-Stage Liver Disease, MRI magnetic resonance imaging, OPTN Organ Procurement and Transplant Network utilization of the T2 exceptions by eliminating some false positive readings for HCC, but will also lead to an increase in biopsy of indeterminate lesions. Longitudinal observation may be considered for smaller indeterminate lesions as these less commonly have washout and a pseudocapsule, and they can be monitored for growth as this is also a characteristic that meets qualifications for HCC diagnosis under the new OPTN system.

Another radiological rating system for liver lesions is LIRADS (Liver Imaging Reporting and Data System (Fig. 1)[3••], developed after a meeting of the American College of Radiology (ACR) in 2008 to address the need to standardize reporting for diagnosing HCC. A major contribution of LI-RADS is its granular definition of radiological criteria for $\mathrm{HCC}$ and standardization for reporting these radiological characteristics to give a probability of certainty of the diagnosis in order to help guide the need for repeat imaging or biopsy. Importantly, input has been sought from radiologists, hepatologists, liver surgeons, and pathologists to help precisely define terminology, create an illustrative atlas along with a the OPTN criteria suggests that these changes will improve 
diagnostic algorithm, and provide guidance for utilization of imaging. Prospective validation studies to establish HCC likelihood according to LI-RADS categories are not yet available but retrospective analysis of MRI has been performed [4]. Hopefully, this system that encompasses all liver lesions will prove helpful for all clinicians caring for patients with liver masses. Whether LI-RADS proves more user-friendly and accurate and is adopted by clinicians and radiologists alike independent of the function of OPTN criteria for defining HCC remains to be determined.

There is an increasing experience with the use of hepatobiliary contrast agents in addition to standard gadolinium for diagnosing HCC and other liver tumors. Their use involves a separate injection of the hepatobiliary contrast agent and scanning following imaging with standard gadolinium-based agents. In an interesting study, Matsuda et al. [5] examined 147 patients undergoing hepatic resection for HCC and looked at whether the pre-operative imaging with gadoxetic acid-enhanced MRI (EOB-MRI) and simultaneous treatment of any early HCC (eHCC) by resection or ablation improved prognosis for patients following their hepatic resection. Of the 147 consecutive patients undergoing their first resection for HCC, 77 received EOB-MRI before resection. Additional treatments were more frequent in EOBMRI patients. Recurrence-free survival was similar at 1 year ( 81.4 vs. $82.1 \%$ ) but improved with EOB-MRI at 3 and 5 years (62.6 and $48.7 \%$ vs. 41.5 and $25.5 \%$, respectively, $p<0.01)$. Though there was a trend towards improved overall survival with EOB-MRI at all timepoints, the result was not statistically significant (1-, 3-, and 5-year survival: 98.7, 90.7, and $80.8 \%$ vs. $97.0,86.3$, and $72.4 \%$, respectively, $p=0.38$ ). Further study on larger numbers of patients is needed before we adopt this technique. However, the finding of improved recurrence-free survival using EOB-MRI argues for the ability of this technique to detect eHCC that later progress to HCC.

The histologic grade of HCC correlates with tumor aggressiveness, and therefore methods to define tumor grade noninvasively add to our ability to predict HCC behavior. Woo et al. [6] compared intravoxel incoherent motion (IVIM)derived parameters, a novel technique to acquire and view MRI images, , with apparent diffusion coefficient (ADC) and correlated this with the histologic grade of HCC. IVIM was shown to be an improvement upon ADC alone as it separates pure diffusion characteristics from 'pseudodiffusion' created by blood flow and microvascular changes that occur with increases in HCC grade. Two radiologists and two pathologists retrospectively evaluated the relationship between IVIMderived parameters and arterial enhancement degree to differentiate between histologic low-grade tumor (grade 1 and 2) and high-grade HCC (grade 3 and 4) in 42 patients that had surgically treated HCC. The enhancement degree, percentage of arterial enhancement of HCC, and histologic grade were analyzed, generating receiver operating characteristic (ROC) analysis of discrimination between low-grade and high-grade HCC for IVIM-derived diffusion coefficient (D) and ADC values. IVIM-derived diffusion coefficient values of $\mathrm{HCC}$ had better diagnostic performance than ADC values in differentiating high-grade $\mathrm{HCC}$ from low-grade $\mathrm{HCC}(0.838$ vs. 0.728 ; $p=0.026$ ), and significant correlation was observed between perfusion fraction and the percentage of arterial enhancement $(\mathrm{r}=0.621, p<0.0001)$. Therefore, this technique may add to current imaging to help predict tumor grade and behavior. Study of IVIM in larger numbers of patients and the increased accuracy of IVIM-derived parameters is necessary before this can be adopted.

Another study by Kakite et al. [7] prospectively looked at the reproducibility of IVIM technology for HCC. In 11 patients, IVIM diffusion weighted imaging (DWI) was compared with ADC using a 3 Tesla (3 T) magnet to identify HCC. Not all parameters were found to be reliably reproducible, but there was good reproducibility for the true diffusion coefficient and $\mathrm{ADC}$ for HCC and liver parenchyma, though diffusion was more reproducible in liver parenchyma. These data argue for the need for more experience with these techniques to achieve higher reproducibility before their universal adoption.

Though contrast images are the standard for non-invasively diagnosing HCC (see discussion on OPTN and LI-RADS above), the resolution of non-contrast studies has improved and it is possible to view the non-contrast MRI studies and still predict the presence of HCC with high probability. The use of $3 \mathrm{~T}$ non-contrast MRI (T1- and T2-weighted images) and diffusion-weighted images were compared by Kim et al. [8] with the diagnostic performance of standard-contrast MRI (EOB-MRI and non-contrast MRI) for detecting hepatic malignancies, HCC, and cholangiocarcinoma. This study included 135 patients with chronic liver disease with histologically confirmed HCC $(n=136)$ and cholangiocarcinomas $(n=12)$, 34 with benign lesions $(\leq 2.0 \mathrm{~cm})$, and patients with cirrhosis but no focal liver lesion $(n=22)$. Imaging results were analyzed independently by three observers to detect liver malignancies using ROC analysis. Using pooled data, they found that the sensitivity of the standard combined contrast and noncontrast MRI (mean $94.8 \%$ ) was higher than the non-contrast MRI (mean $91.7 \%)(p=0.001)$, but specificity was equivalent (78.6 vs. $77.5 \%$ ). Non-contrast MRI with DWI compared favorably with the combined EOB-MRI and non-contrast MRI for detecting HCC and cholangiocarcinoma and in differentiating them from benign lesions in patients with chronic liver disease. These data have implications for the use of MRI for screening and surveillance of liver cancers in patients with liver disease as there is the potential for reducing the use of contrast and reducing the time for performing MRI studies, factors that contribute to the cost and tolerability of MRI.

The overall utility of the use of DWI for the diagnosis of HCC in patients with chronic liver disease without the use of contrast to perform MRI was examined by Wu et al. [9] using 
Fig. 1 LI-RADS (Liver Imaging Reporting and Data System) radiological rating system for liver lesions (with permission from the American College of Radiology [2]). HCC hepatocellular carcinoma

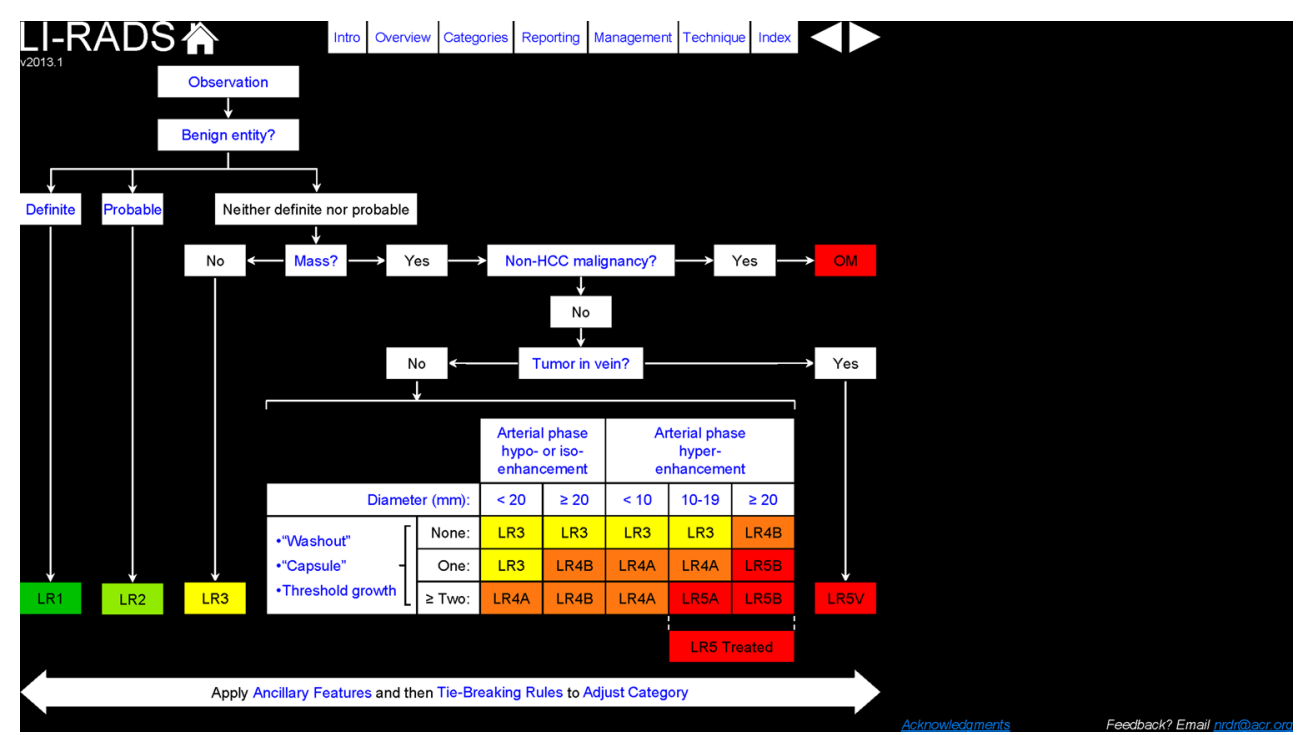

meta-analysis of published data from January 2000 to April 2012 that included nine studies with 476 patients. DWI sensitivity was $81 \%$ (95\% CI 67-90) and specificity was $89 \%$ (95\% CI 76-95). The area under the concentration-time curve (AUC) of the ROC was 0.92 (95\% CI 0.89-0.94). Though no major differences were found between contrastenhanced MRI and DWI MRI, when DWI was combined with contrast-enhanced studies there was a statistically significant higher pooled sensitivity than DWI by itself (93 vs. $73 \%$, $p<0.05)$. These data suggest that while DWI can provide good HCC detection, combining contrast-enhanced and DWI MRI can further improve diagnostic accuracy.

There are situations where HCC may arise in non-cirrhotic liver, e.g., in patients with chronic hepatitis B or non-alcoholic steatohepatitis. Di Martino et al. [10] examined whether characteristics of HCC in non-cirrhotic liver on MRI with gadolinium-based contrast and multi-detector computed tomography (CT) imaging were similar to cirrhotic liver with respect to $\mathrm{HCC}$ size, presence of tumor capsule, necrosis, hemorrhage, fat, calcification, and vascular involvement. Two observers retrospectively compared imaging patterns for 32 HCCs in 30 non-cirrhotic patients with HCC in cirrhotic patients. In non-cirrhotic patients, they found typical imaging characteristics in $84 \%$ of HCC on CT and $87.5 \%$ on MRI, suggesting imaging characteristics are similar for $\mathrm{HCC}$ on dynamic imaging of the liver in non-cirrhotic and cirrhotic liver.

\section{Assessment of HCC Treatment}

As patients undergo treatments for HCC while awaiting liver transplant, there is a specific need to assess the efficacy of treatment and determine the need for tumor re-treatment. As HCC increases the blood flow supplied mainly from the hepatic artery, and since treatments such as arterial embolization (with and without chemotherapy) and other systemic agents that block endothelial vascular growth can diminish tumor blood supply, the major focus for determining treatment efficacy is on estimating changes in HCC perfusion. Taouli et al. [11] prospectively studied 26 patients with 33 HCC lesions (16 untreated and 17 treated with chemoembolization; mean size $3.9 \mathrm{~cm}$; range $1.1-12.6 \mathrm{~cm}$ ) using dynamic contrast-enhanced MRI (DCE-MRI) for perfusion quantification of HCC and surrounding liver. When compared with liver parenchyma, HCC showed statistically significant higher arterial hepatic blood flow and arterial fraction and lower distribution volume and portal venous hepatic blood flow with no difference in mean transit time. Untreated HCC had a higher arterial fraction and lower portal venous hepatic blood flow value than treated (chemoembolized) HCC $(p<0.04)$. Therefore, quantification of changes in perfusion are possible after treatment with chemoembolization and may be useful along with other parameters for assessing tumor response to treatment.

Sorafenib is the only systemic chemotherapeutic agent approved for treating $\mathrm{HCC}$, but assessing response to this agent is challenging. There are several standard oncological methods for assessing tumor treatment response such as monitoring for decreases in alpha fetoprotein (AFP) [12], and different criteria have been put forth by liver societies: Response Evaluation Criteria in Solid Tumors (RECIST) 1.1, European Association for the Study of the Liver (EASL), modified RECIST (mRECIST), and Choi's criteria, which monitors change in tumor density and arterial-enhancing tumor volume variations. These methods for assessing tumor response were compared in sorafenib-treated patients $(n=22)$, showing that response criteria and volume measurements were reproducible $(\mathrm{k}>$ 0.80). The disease control rate was $40.9 \%$ by EASL and mRECIST, and $27.3 \%$ by RECIST 1.1 . A greater than $15 \%$ decrease in tumor density was observed in nine patients $(40.9 \%)$. The mean volume ratio was $1.73 \pm 2.12$ and the mean 
AFP ratio was $14 \pm 37$. The 1 -year survival rate was $65.9 \%$. The volume ratio was shown to be the only predictive factor for survival, with 1-year cumulative survival rates of $90 \%$ for volume ratios $\leq 1.1$ and only $45.4 \%$ survival for volume ratios $>1.1(p=0.04)$. Therefore, tumor volume measurements may be an early predictive marker of HCC response to sorafenib treatment. We can only speculate that an increased predictive value of tumor response and survival (in patients matched for their Childs status) may be possible when volume estimates are combined with estimates of tumor perfusion.

Another method to attempt to improve the accuracy of tumor treatment and assessing treatment efficacy is threedimensional (3D) registration of images before and after tumor ablation by radiofrequency ablation (RFA). Sakakibara et al. [13] previously used side-by-side comparisons of preand post-RFA imaging studies in 139 HCC nodules in 84 patients, and retrospectively performed $3 \mathrm{D}$ registration on the imaging from the same studies. They found that histological grade at the margin and proximity to blood vessels $\geq 3 \mathrm{~mm}$ were predictors of tumor recurrence. Recurrence $(n=22)$ occurred more frequently and was more easily detected using $3 \mathrm{D}$ registration when there was residual tumor $(n=13)$ or no margin from tumor and treated area $(n=6)$. These data suggest that $3 \mathrm{D}$ registration is more useful for assessing ablative margins than standard side-by-side comparison of pre- and post-treatment imaging, and that no margin after treatment and proximity to blood vessels $>3 \mathrm{~mm}$ are associated with high tumor recurrence rates following RFA.

Another method to assess tumor treatment by chemoembolization involves imaging to determine the intraarterial perfusion using MRI [transcatheter intra-arterial perfusion (TRIP) MRI]. Wang et al [14] measured tumor perfusion changes during transarterial chemoembolization and looked at its impact on transplant-free survival (TFS) in 51 consecutive patients with unresectable HCC. Patients with $35-85 \%$ intra-procedural tumor area under the time-signal intensity curve reduction $(n=32)$ showed significantly improved median TFS: 16.6 months compared with only 9.3 months ( $p=0.046,95 \%$ CI $0.21-1.00)$ in patients with an area under the time-signal intensity curve reduction outside this range $(n=18)$. Furthermore, cumulative TFS rates over time (1, 2, and 5 years) in the $35-85 \%$ and the $<35 \%$ tumor perfusion reduction groups after chemoembolization were $66.4,42.2$, and $28.2 \%$ versus $33.8,16.9$, and $0 \%$, respectively. Therefore, TRIP MRI-measured tumor perfusion reduction may be useful as an intra-procedural imaging biomarker during chemoembolization.

\section{Contrast-Enhanced Ultrasound}

Contrast-enhanced ultrasound (CEUS) that can measure not only the tumor size but also contrast agent uptake as a marker of tumor vascularity was studied for use in early detection and tumor response to treatment. A recent meta-analysis by Niu et al. [15•] reviewed 15 studies with 908 cirrhotic patients with 1,032 small hepatic nodules detected using CEUS that were histologically confirmed as HCC with liver biopsy after imaging. The pooled sensitivity of CEUS detection was 0.81 (95\% CI $0.78-0.85)$ with specificity of 0.86 (95\% CI 0.82 0.89 ) and a diagnostic odds ratio of 37.07 (95\% CI 27.78 55.44). Though the number of lesions was a major source of heterogeneity, CEUS may be a promising cost-efficient method for early detection of HCC in comparison to contrast CT or MRI [16]. Issues related to more widespread adoption of this technique include technical proficiency and reproducibility in performance of the study, and linking radiologists and technicians closely to review the quality of the studies in real time.

There are also additional data showing that CEUS can be used to assess HCC treatment using perfusion as a marker of response, as was noted above for MRI. Frampas et al. [17] investigated the use of CEUS in patients with advanced HCC treated with sorafenib, comparing CEUS results with perfusion CT imaging. For CEUS, quantitative perfusion measurements were obtained from time-intensity curses with total AUC, AUC during wash-in (AUCWI), and AUC during wash-out (AUCWO) measurements. Nineteen patients had baseline imaging with contrast-enhanced CT and CEUS at month 1 and month 2, at which the time to progression was assessed. Those with an AUC decrease $>40 \%(n=6)$ during follow-up did not have progression of disease at month 2 by RECIST criteria, while four of five patients in whom AUC decreased $\leq 40 \%$ had progression. However, change in perfusion indices did not differentiate progressors from nonprogressors using perfusion $\mathrm{CT}$ imaging. This suggested an earlier assessment of tumor response was possible with CEUS; however, the impact of the study was limited by the small number of patients examined.

CEUS was also compared with contrast-enhanced CT (CECT) for recurrent HCC in the follow-up assessment of percutaneous ablation therapy. Zheng et al. [18] followed 141 patients with HCC who had percutaneous tumor ablation, which included mostly RFA $(n=83)$, percutaneous ethanol ablation (EA) $(n=29)$, or combination therapy of RFA and EA $(n=26)$, and microwave ablation $(n=3)$. These patients were followed with both CEUS and CECT and were assessed for local tumor progression (LTP), defined by recurrence inside or adjacent to the treated site, and also assessed for new lesions. The sensitivity of CEUS in detecting LTP and new intrahepatic lesions was significantly low, at 67.5 and $77.7 \%$, respectively. A similar finding was seen in a study [19] that compared CEUS with positron emission tomography (PET) CT with fluorine-18 fluorodeoxyglucose $\left({ }^{18} \mathrm{~F}-\mathrm{FDG}-\right.$ $\mathrm{PET} / \mathrm{CT}$ ) in patients who had surgical resection and/or RFA. CEUS also showed a lower sensitivity for detecting intrahepatic HCC with sensitivity of $56.7 \%$ (vs. $96.7 \%$ with 
PET CT). These studies may be explained by the intrinsic limitation of ultrasound techniques in detecting lesions at the dome of the liver, or obscuration by gas from gastrointestinal tract or lung. Given the technical limitations for CEUS in detection and performance, it is likely this technique will remain complementary to MRI and CT evaluation of $\mathrm{HCC}$ and its treatment.

\section{Living Donor Liver Transplant}

Liver imaging plays an important role in planning for LDLT by permitting non-invasive estimation of volume of liver segments and permitting identification of vascular and biliary anatomy. Other uses for imaging in living donor evaluation include the estimation of fat content of liver tissue and evaluation for biliary leaks post-operatively. The following discussion highlights new studies in imaging as it relates to LDLT.

Radiographic features of living donor candidates are critical for determining their eligibility to donate. Hahn et al. [20•] reviewed the radiographic features of 159 consecutive candidates for living liver donation using multi-detector CT (MDCT) cholangiography and angiography. Two of the patients (1\%) that proceeded to donation were cleared pre-operatively by MDCT imaging, but intraoperative findings led to cancellation of the donor surgery. Of the 61 candidates excluded from liver donation on the basis of imaging findings, inadequate liver volume was the most common reason for exclusion $(n=40$ / $61 ; 66 \%)$. The reasons for exclusion for donation were vascular or biliary variants (for right- and left-lobe donation) $(n=14)$, steatosis $(n=5)$, and renal cell carcinoma being detected $(n=2)$. Given the high frequency of exclusion for liver volume, it is likely that a cost-effective method for screening living liver donors may involve rapid determination of segmental liver volumes prior to more extensive studies.

One area of concern for selection of living donor candidates is the presence of steatosis in potential donor candidate livers. Most often, the presence of steatosis correlates with elevated body mass index (BMI); however, quantification of steatosis is important to accurately assess liver volume. Increased steatosis inflates the liver volume relative to the true amount of parenchymal tissue and can change the BMI ratio. Apart from biopsy and direct visualization, technical advances in MRI using proton density fat fraction (PDFF) measurements permitted better quantification of liver fat content in patients with non-alcoholic fatty liver disease (NAFLD) that compared favorably with liver biopsy findings [21]. When fibrosis was absent, there was an excellent correlation between PDFF and liver biopsy $(r=0.82)$ and discrimination between moderate and severe steatosis and mild or no steatosis gave an AUC of 0.95 . With fibrosis present, correlation was reduced $(r=0.60)$. Therefore, in donors who would mostly be presumed to be without any hepatic fibrosis, PDFF could be a useful tool to look for and quantify hepatic steatosis. This may be especially useful if a potential donor with steatosis is asked to lose weight as it allows non-invasive estimation of steatosis that can be repeated as often as necessary.

\section{Fibrosis Assessment in Liver Grafts}

Allograft dysfunction is a major post-transplant problem in LDLT and early detection of graft dysfunction or fibrosis is very useful. Graft fibrosis is difficult to detect early, as it can be present without abnormality of liver biochemistry results. Of the multiple elasticity imaging techniques that have been developed over the years, acoustic radiation force impulse elastography (ARFI) is a new elastography method developed to evaluate tissue stiffness, similar to transient elastography. Only a few studies have looked into the use of ARFI in the post-liver transplant population. Compared with transient elastography, ARFI can be integrated into a conventional ultrasound system and performed during a standard examination of the liver. It can also be used under B-mode imaging and can detect nearby structures such as blood vessels under realtime imaging. ARFI can also be used from the midline of the upper abdomen as well as the right intercostal space, which may be more appropriate for LDLT patients who received leftlobe grafts.

Liao et al. [22] used ARFI to evaluate liver fibrosis in LDLT patients. LDLT patients $(n=57)$ underwent both ARFI liver stiffness measurements (LSM) as well as liver biopsy due to graft dysfunction [defined as aspartate aminotransferase and alanine aminotransferase (ALT) $>100 \mathrm{IU} / \mathrm{dL}$ or persistent hyperbilirubinemia]. AFRI LSM values for liver fibrosis showed a significant linear correlation with the pathological fibrosis stage. LSM had a specificity of 83.6 and $92.9 \%$ for fibrosis scores of $\mathrm{F} \geq 2$ and $\geq 3$, respectively, with cutoff values of $1.81 \mathrm{~m} / \mathrm{s}$ for F2 and $2.33 \mathrm{~m} / \mathrm{s}$ for F3. In this study, there was also a distinction between F0 and F1, with high sensitivity of $95.5 \%$, suggesting early fibrosis detection, which was not revealed in an earlier study [23•]. ARFI was again used for assessment of graft fibrosis in a pediatric LDLT population where graft fibrosis is more common on protocol biopsy. Once again, ARFI LSM showed a good correlation with significant graft fibrosis defined as F2 or higher, with areas under the ROC curve (AUROCs) of $0.760(p=0.005)$ and 0.849 $(p<0.001)$ for the midline and intercostal values, respectively. Although both studies are limited by small size, they suggest that the measurement of liver stiffness by ARFI imaging is a useful alternative to protocol biopsy, or can be even considered as a bridge between biopsies.

Magnetic resonance elastography (MRE) is an MRI-based method for quantitatively imaging the direct consequence of liver fibrosis and can assess larger regions of the liver rather 
than localized spot measurements through an ultrasoundbased technique [24]. Crespo et al. [25] conducted a prospective study on 54 liver transplant patients with hepatitis $\mathrm{C}$ virus who underwent both protocol liver biopsy and MRE. Significant correlation was seen between histologic fibrosis and shear stiffness $\left(\mathrm{R}^{2}=0.588, p<0.0001\right)$, with $91 \%$ sensitivity and $72 \%$ specificity in differentiating fibrosis grade $\geq 3$ from 0 to 1 , using a cutoff value of $3.5 \mathrm{kPa}$. However, the limitations of MRE should be considered in livers with high iron deposition, which was also seen in this study. Another confounder of accurate LSM with MRE could be hepatitis activity with inflammation seen on biopsy, where an independent relationship was seen between ALT and liver stiffness, leading to a false positive rate of $46 \%$ for cirrhosis [26]. This method will continue to be developed; however, use of MRI for this purpose may be more useful for clinical research studies and less practical for clinical use than elastography by sonography given the need for use of MRI time and its lack of portability to the clinical site where real-time study performance is possible for sonographic techniques.

\section{Conclusion}

The many advances during this year in imaging techniques and their application to the evaluation of liver structure and anatomy, tumor detection, and the evaluation of liver tumor treatment have already impacted on our care for liver transplant patients. Further refinement and testing of these techniques will pave the way for their adoption in the clinic.

\section{Compliance with Ethics Guidelines}

Conflict of Interest A.K. Kim declares no conflicts of interest.

M.L. Schilsky has received consultancy fees from Wilson's Therapeutics.

Human and Animal Rights and Informed Consent This article does not contain any studies with human or animal subjects performed by any of the authors.

\section{References}

Papers of particular interest, published recently, have been highlighted as:

- Of importance

•. Of major importance

1. OPTN policy. 13 Oct. 3.6: Allocation of livers. http://optn. transplant.hrsa.gov/PoliciesandBylaws2/policies/pdfs/policy_8. pdf. Accessed 30 Aug 2014.

2.• Fowler KJ, Karimova EJ, Arauz AR, Saad NE, Brunt EM, Chapman WC, et al. Validation of Organ Procurement and Transplant Network (OPTN)/United Network for Organ Sharing
(UNOS) criteria for imaging diagnosis of hepatocellular carcinoma. Transplantation. 2013;95(12):1506-11. This study supports the current use of OPTN criteria for HCC diagnosis based on pretransplant MRI by demonstrating a low rate of patients with $T 2$ tumor burden without OPTN criteria radiological evidence for HCC. This suggests an improved utilization of the T2 exceptions will occur by eliminating some false positive readings for HCC.

3.• American College of Radiology. Liver Imaging Reporting and Data System version 2013.1. Accessed June 2014, from http://www.acr. org/Quality-Safety/Resources/LIRADS/. Protocol recommended by ACR to standardize reporting for HCC diagnosis as well as benign liver lesions.

4. Petruzzi N, Mitchell D, Guglielmo F, et al. Hepatocellular carcinoma likelihood on MRI exams: evaluation of a standardized categorization system. Acad Radiol. 2013;20:694-8.

5. Matsuda M, Ichikawa T, Amemiya H, Maki A, Watanabe M, Kawaida H, Kono H, Sano K, Motosugi U, Fujii H. Preoperative gadoxetic acid-enhanced MRI and simultaneous treatment of early hepatocellular carcinoma prolonged recurrence-free survival of progressed hepatocellular carcinoma patients after hepatic resection. HPB Surg. 2014:641685.

6. Woo S, Lee JM, Yoon JH, Joo I, Han JK, Choi BI. Intravoxel incoherent motion diffusion-weighted MR imaging of hepatocellular carcinoma: correlation with enhancement degree and histologic grade. Radiology. 2014;270:758-67.

7. Kakite S, Dyvorne H, Besa C, Cooper N, Facciuto M, Donnerhack C, Taouli B. Hepatocellular carcinoma: short-term reproducibility of apparent diffusion coefficient and intravoxel incoherent motion parameters at 3.0T. J Magn Reson Imaging.

8. Kim YK, Kim YK, Park HJ, Park MJ, Lee WJ, Choi D. Noncontrast MRI with diffusion-weighted imaging as the sole imaging modality for detecting liver malignancy in patients with high risk for hepatocellular carcinoma. Magn Reson Imaging. 2014;32:610-8.

9. Wu LM, Xu JR, Lu Q, Hua J, Chen J, Hu J. A pooled analysis of diffusion-weighted imaging in the diagnosis of hepatocellular carcinoma in chronic liver diseases. J Gastroenterol Hepatol. 2013;28: 227-34.

10. Di Martino M, Saba L, Bosco S, Rossi M, Miles KA, Di Miscio R, et al. Hepatocellular carcinoma (HCC) in noncirrhotic liver: clinical, radiological and pathological findings. Eur Radiol. 2014;24(7):1446-54. doi:10.1007/s00330014-3173-2.

11. Taouli B, Johnson RS, Hajdu CH, Oei MT, Merad M, Yee H, et al. Hepatocellular carcinoma: perfusion quantification with dynamic contrast-enhanced MRI. AJR Am J Roentgenol. 2013;201:795800 .

12. Bargellini I, Scionti A, Mismas V, Masi G, Vivaldi C, Bartolozzi C, et al. Identification of responders to sorafenib in hepatocellular carcinoma: is tumor volume measurement the way forward? Oncology. 2014;86:191-8.

13. Sakakibara M, Ohkawa K, Katayama K, Imanaka K, Ishihara A, Hasegawa N, et al. Three-dimensional registration of images obtained before and after radiofrequency ablation of hepatocellular carcinoma to assess treatment adequacy. AJR Am J Roentgenol. 2014;202:W487-95.

14. Wang D, Gaba RC, Jin B, Lewandowski RJ, Riaz A, Memon K, et al. Perfusion reduction at transcatheter intraarterial perfusion MR imaging: a promising intraprocedural biomarker to predict transplant-free survival during chemoembolization of hepatocellular carcinoma. Radiology. 2014;272:587-97.

15. Niu Y, Huang T, Lian F, Li F. Contrast-enhanced ultrasonography for the diagnosis of small hepatocellular carcinoma: a meta-analysis and meta-regression analysis. Tumour Biol. 2013;34(6):3667-74. This study shows promising data on the use of CEUS for detecting small HCC. 
16. Sirli R, Sporea I, Martie A, Popescu A, Dănilă M. Contrast enhanced ultrasound in focal liver lesions-a cost efficiency study. Med Ultrason. 2010;12(4):280-5.

17. Frampas E, Lassau N, Zappa M, Vullierme MP, Koscielny S, Vilgrain V. Advanced Hepatocellular Carcinoma: early evaluation of response to targeted therapy and prognostic value of perfusion $\mathrm{CT}$ and dynamic contrast enhanced-ultrasound. Preliminary results. Eur J Radiol. 2013;82(5):e205-11.

18. Zheng SG, Xu HX, Lu MD, Xie XY, Xu ZF, Liu GJ, et al. Role of contrast-enhanced ultrasound in follow-up assessment after ablation for hepatocellular carcinoma. World J Gastroenterol. 2013;19(6):855-65.

19. Wang XY, Chen D, Zhang XS, Chen ZF, Hu AB. Value of ${ }^{18}$ F-FDG$\mathrm{PET} / \mathrm{CT}$ in the detection of recurrent hepatocellular carcinoma after hepatectomy or radiofrequency ablation: a comparative study with contrast-enhanced ultrasound. J Dig Dis. 2013;14(8):433-8.

20. Hahn LD, Emre SH, Israel GM. Radiographic features of potential donor livers that precluded donation. AJR Am J Roentgenol. 2014;202:W343-8. Retrospective data of CT angiography and cholangiography findings of potential living donors and factors that preclude donation. Inadequate liver volume was the most common reason for exclusion in $60 \%$ of candidates.

21. Idilman IS, Aniktar H, Idilman R, Kabacam G, Savas B, Elhan A, et al. Hepatic steatosis: quantification by proton density fat fraction with MR imaging versus liver biopsy. Radiology. 2013;267:767-75.

22. Liao CC, Chen TY, Tsang LC, Ou SY, Yu CY, Hsu HW, et al. The acoustic radiation force impulse elastography evaluation of liver fibrosis in posttransplantation dysfunction of living donor liver transplantation. Transplant Proc. 2014;46(3):876-9.

23. Crespo G, Lens S, Gambato M, Carrión JA, Mariño Z, Londoño $\mathrm{MC}$, et al. Liver stiffness 1 year after transplantation predicts clinical outcomes in patients with recurrent hepatitis C. Am J Transplant. 2014;14(2):375-83. This is a prospective study on post-liver transplant population, evaluating the use of MRE for assessment of fibrosis.

24. Venkatesh SK, Yin M, Ehman RL. Magnetic resonance elastography of liver: clinical applications. J Comput Assist Tomogr. 2013;37(6):887-96.

25. Crespo S, Bridges M, Nakhleh R, McPhail A, Pungpapong S, Keaveny AP. Non-invasive assessment of liver fibrosis using magnetic resonance elastography in liver transplant recipients with hepatitis C. Clin Transplant. 2013;27(5):652-8.

26. Ichikawa S, Motosugi U, Nakazawa T, Morisaka H, Sano K, Ichikawa T, Enomoto N, Matsuda M, Fujii H, Onishi H. Hepatitis activity should be considered a confounder of liver stiffness measured with MR elastography. J Magn Reson Imaging. 